\title{
La construcción de los derechos sociales desde la privación legal de la libertad en América Latina*
}

\author{
Building Social Rights for Individuals Legally Deprived \\ of their Liberty in Latin America
}

Cristina Rumbo Bonfil ${ }^{* *}$

Fecha de recepción: 5 de mayo del 2020 Fecha de aprobación: 25 de mayo del 2020

\section{Resumen}

La identificación y construcción de los derechos sociales aplicables al derecho ejecutivo penal obedecen a la falta de una tradición jurídica que omite y restringe la aplicación efectiva de la protección de los derechos de las personas privadas de la libertad. No solo se trata de las violaciones en materia de derechos humanos o de su inobservancia, sino de la omisión normativa de todos los derechos que no son privativos de la conducta antijurídica y que no corresponden a una aplicación práctica que coadyuve a la configuración del cumplimiento de la función ius puniendi del Estado y de la pena en sí misma, y que es la reinserción social.

Palabras clave: derechos humanos, derechos sociales, privación legal de la libertad, sistema penitenciario, crisis, reforma, persona.

\footnotetext{
Artículo resultado de investigación y desarrollado en cooperación con el grupo de investigación Red Internacional de Política Criminal Sistémica Extrema Ratio UN de la Universidad Nacional de Colombia, reconocido y clasificado en A1 por Minciencias (2018).

Citar como: Rumbo Bonfil, C. (2020). La construcción de los derechos sociales desde la privación legal de la libertad en América Latina. Via Inveniendi et Iudicandi, 15(2), 165-186. DoI: https://doi. org/10.15332/19090528/6246

** Doctora en Derecho y Globalización por la Universidad Autónoma del Estado de Morelos (México); coinvestigadora del grupo de investigación Red Internacional de Política Criminal Sistémica Extrema Ratio UN de la Universidad Nacional de Colombia. Correo: lic.cristinarb@gmail.com / cristina.rumbo@morelos. gob.mx ORCID: 0000-0002-6724-5147
} 


\begin{abstract}
The identification and construction of social rights applicable to criminal executive law are conditioned by the faults of a legal tradition that omits and restricts the effective application of the rights of individuals legally deprived of their liberty. The issue is not only related to human rights violations or their non-observance, but also about to the normative omission of all rights that are not exclusive of unlawful conduct and that do not correspond to a practical application that contributes to the configuration of the fulfillment of the ius puniendi function of the State and of the penalty itself, which is social reintegration.
\end{abstract}

Keywords: human rights, social rights, legal deprivation of liberty, prison system, crisis, reform, person. 


\section{INTRODUCCIÓN}

Este artículo pretende abordar el estudio e identificación de los derechos sociales en América Latina y su construcción a partir de una tutela judicial efectiva que permita su incorporación en el derecho ejecutivo penal, estrictamente en el reconocimiento de los derechos sociales de las personas privadas legalmente de la libertad. ¿Es posible que estas personas puedan acceder al reconocimiento e incorporación de los derechos sociales durante dicha privación? Es posible centrar el debate hacía la posibilidad de reconocer la construcción de los derechos sociales en las personas privadas de la libertad; en qué momento es posible su asignación y derecho legítimo, cuando la construcción de los derechos en sí mismos es todavía una cuenta pendiente.

El liberalismo político y económico ha plagado el derecho interno de la incorporación de nuevas y progresivas generaciones de derechos que el campo de lo práctico y lo factible poco hace por los grupos sociales más desfavorecidos. Los derechos sociales deben su desarrollo a un profundo sentido igualitario, que propugna por su ejercicio sin restricción alguna. Incluso, el Estado mexicano forma parte de una larga tradición constitucional frente al reconocimiento de los derechos y garantías sociales, a partir de los cuales se le dio a la Carta Magna de 1917 el mote de "la primera Constitución garantista del mundo"; lo que quedó rebasado ante la tardía incorporación de nuestra constitución y de nuestro sistema jurídico a la corriente ius filosófica del garantismo, imperante en el mundo a partir del sigloxx, al término de la segunda guerra mundial.

Los derechos sociales representan una contradicción de carácter jurídico en México y en la gran mayoría de Estados latinoamericanos:

[...] es posible identificar a los derechos sociales con derechos prestacionales, esto es, con aquellos derechos que, en lugar de satisfacerse mediante una abstención del sujeto obligado (el Estado principalmente) requieren por su parte de una acción positiva que se traduce normalmente en la prestación de un bien o servicio. (Cruz Parcero, 2000, pp. 90-91)

Ello supone la problemática de su instauración en el entorno social a las clases menos favorecidas, que encuentran en la aplicación de los derechos sociales potestades estatales 
que, lejos de ser irreductibles, conllevan ineficaces políticas públicas para protegerlos. Así, derechos sociales como los del empleo, la vivienda, la educación y la salud, además de los derechos a un entorno saludable y a la alimentación (Organización de las Naciones Unidas, ONU, 2020) constituyen retos estatales que se convierten en invisibles y de imposible alcance.

La estructura del cumplimiento de los derechos sociales representa un reto absolutamente complicado, que se reduce a brindar los mínimos indispensables que el Estado de bienestar (llámese ahora Estado de derecho) logra —o quiere— satisfacer. Por ello, el hecho de comenzar a relatar cuántos grupos de minorías (y de qué calidad) son los que pueden aspirar a un cumplimiento mucho más eficaz de sus derechos fundamentales sería una tarea mucho más práctica para el ejercicio de políticas públicas — y, en cierto grado, de políticas clientelistas que propicien el cumplimiento de los derechos políticos a partir de los llamados derechos prestacionales.

Frente a esto, entendemos que la tarea del Estado es la de crear una política criminal sistémica (Huertas Díaz, 2019) o mecanismos que permitan proteger a los distintos grupos sociales, destacando por principio a los grupos considerados más débiles o que, por su condición, enfrentan mayores dificultades para acceder a derechos (grupos vulnerables). Robert Alexy (1993, p. 449) describe los derechos como un acto positivo del Estado; en ese sentido, enmarca los derechos de protección (individual) frente al Estado, lo que presupone la obligación que este tiene de proteger al individuo frente a intervenciones de terceros (Cruz Parcero, 2012, pp. 76-77). Sin embargo, ¿̨cómo identificamos cuáles grupos o individuos deben ser protegidos en primer lugar?

Con este supuesto, tendríamos que elaborar una larga lista de grupos sociales que, siguiendo a Alexy y el principio de proporcionalidad, deben o pueden sujetarse a la aplicación de un derecho primero que, en grado de urgencia, deba ser aplicado sobre un derecho segundo. Frente a este grado de urgencia, existen muchos grupos sociales que reclaman la observancia inmediata de sus derechos fundamentales, pues hoy en día la aplicación de los presupuestos constitucionales que reconocen la aplicación de esos derechos se encuentra lamentablemente en franca agonía. Existen, bajo el fracaso del Estado democrático, miles 
de injusticias y desigualdades que se han acrecentado irónicamente con la adopción del modelo garantista; y no porque el modelo no exija o permita su observación, sino porque sencillamente ha hecho más visibles las carencias de los Estados y ha recrudecido la lucha inalcanzable por la observancia de los derechos humanos.

Bobbio incluso señala que "la especificación de los derechos ha tenido un paso gradual [...] el paso se ha producido del hombre genérico [...] al hombre en específico, o sea, en la especificidad de sus diversos status sociales" (1991, pp. 109-114). En este sentido, Bobbio nos obliga a repensar la postura que asume el Estado frente a esos diversos grupos sociales, pues si bien una de las características que define los derechos fundamentales es su progresividad, la referencia de Bobbio se dirige hacía el surgimiento de esas pequeñas minorías que cada día se hacen más notorias frente a los grandes grupos sociales y que han reclamado de forma mucho más fehaciente no solo el reconocimiento de su identidad como minorías, sino su restitución como sujetos de derechos.

Dentro de dicho reconocimiento de esos diversos grupos sociales, se cuentan principios constitucionales que, desde los derechos prestacionales, adquieren potestades estatales que incluyen desde bienes y servicios hasta derechos ya conocidos, como los de la educación, el empleo y la vivienda, entre otros, y que generalmente se asumen como derechos para los grupos menos favorecidos, pues se debe proteger a los más débiles de los más fuertes.

Sin embargo, hablar de personas privadas de la libertad presupone preceptos distintos: ¡en qué grupo de minorías deben ser incluidos los que delinquen? Y, más aun, como sujetos expuestos a la violación sistemática de sus derechos, ¿deben ser considerados como un grupo que deba adquirir una condición de vulnerabilidad que deba ser protegido bajo la premisa de los principios constitucionales? Su incorporación dentro de este último sistema representa un reto de reconocimiento jurídico y moral, pero, sobre todo, social, pues resulta temerario que el Estado brinde mecanismos de protección a sujetos que hayan dañado y vulnerado el tejido social y que, por tanto, como parte del grupo de los débiles deban ser considerados muy al fondo de una larga lista de prioridades jurídico-sociales. 


\section{Metodología}

La metodología es conocida coloquialmente como el conjunto de pasos sistemáticos que nos permiten conducir un trabajo de investigación, con la firme finalidad de no perderse; y el propósito del método consiste en que, por medio de lo que conocemos como ciencia jurídica, se tenga acceso a un conocimiento nuevo. Así lo señala Javier Hervada (2000):

La ciencia jurídica se caracteriza por ser un conocimiento fenoménico. Por ser ciencia se pregunta por las causas, pero no se dirige a la captación de las causas íntimas, que explican en su integridad la realidad jurídica, sino tan solo las causas próximas y aparentes. (p. 197)

Se dice también que en el área del derecho no existe un método definitivo y que todo parte del ya conocido método de investigación jurídica. La generación del conocimiento parte — como lo dice Aristóteles — de la intuición, pero se necesita un carácter reflexivo e interpretativo que nos permita dilucidar dónde existe una verdadera problemática a la que es posible darle respuesta o analizar bajo un enfoque distinto. La metodología permitirá, entonces, conducir a ese investigador en la obtención de dichas respuestas, creando al final de ese camino un nuevo conocimiento. Este siempre dependerá de las nuevas ideas que se le puedan ocurrir al investigador; sin embargo, como lo menciona Sánchez Zorrilla (2011),

[...] la metodología no es capaz de hacer que se produzcan esas nuevas ideas, no nos brinda un recetario para su invención. La metodología solo describe y presenta las estrategias más fructíferas que permiten que estas ideas puedan ser consideradas como nuevo conocimiento. La estrategia y el conjunto de reglas usadas por los científicos se llama método científico". (pp. 317-358)

Si partimos entonces de la idea de que no se necesita una metodología, sino un método científico, de modo específico, tenemos que construir un trabajo que nos conduzca con estricta verdad a la resolución del problema planteado (hipótesis). Pero tenemos claro que, a partir de los métodos de investigación jurídica, podremos tener una aproximación de los "caminos" que habremos de tomar para llegar a esa verdad esperada, pues, como señala 
Sánchez Zorrilla, “cuando hablamos de metodología jurídica, fundamentalmente nos estamos refiriendo al estudio y análisis del procedimiento para poder determinar cuál es la respuesta jurídica para el caso que estamos examinando, aunque, como veremos, incluye también muchos otros aspectos" (p. 340). Pero también existe la posibilidad de que esos "caminos" sean distintos, pues a través de este método científico estudiaremos las posibles variables que frente al problema planteado nos puedan surgir: "La conclusión que se obtendrá será que lo que hacen los juristas involucra una serie de métodos de muy diversa índole, que muchas veces son ignorados en la formación profesional" (Cruz Parcero, 2006, p. 19).

Con ello, tendremos entonces la tarea de "elegir" los métodos de investigación, pues debemos sumarnos al supuesto de que no existe un método único, sino una serie de métodos a partir de los cuales se debe o se pretende abordar un trabajo de investigación con las premisas de ser 1) un trabajo del área de las ciencias sociales y 2) un trabajo con un predominio del valor dogmático-científico. Un trabajo de investigación o artículo requiere, pues, no solo el uso de métodos, sino también de concepciones filosóficas que eleven la discusión de los problemas sociales a un nivel epistémico que produzca un valor verdaderamente científico y que cumpla con aquella primera pregunta que plantean el investigador y, al tiempo, el lector.

Llamemos filosofía científica a la clase de concepciones filosóficas que aceptan el método de la ciencia como la manera que nos permite: a) plantear cuestiones fácticas "razonables"; esto es, preguntas que sean significativas, no triviales, y que probablemente puedan ser respondidas dentro de una teoría existente o concebible y b) probar respuestas probables en todos los campos especiales del conocimiento.

Así, pues, tendremos que para elaborar el presente artículo se utilizaron distintos métodos de investigación. Además de trabajar por medio de las metodologías de investigación jurídica, se emplean dos métodos de investigación conocidos de forma más amplia a través de las ciencias sociales: tanto el método exploratorio como el explicativo ofrecen mecanismos de investigación que acercaran nuestro trabajo a los objetivos planteados.

El método exploratorio "ayuda a familiarizarse con fenómenos desconocidos, obtener información para realizar una investigación más completa en un contexto particular, investigar nuevos problemas, identificar conceptos o variables promisorias, establecer 
prioridades para investigaciones futuras, o sugerir afirmaciones y postulados" (Hernández Sampieri, Fernández Collado y Baptista Lucio, 2014, p. 91). Por su parte, el método explicativo, según estos autores,

[...] está dirigido a responder por las causas de los eventos y fenómenos físicos o sociales. Se enfoca en explicar por qué ocurre un fenómeno y en qué condiciones se manifiesta, o por qué se relacionan dos o más variables $[\ldots]$ Se encuentra más estructurado que los demás alcances (de hecho implica los propósitos de estos); además de que proporciona un sentido de entendimiento del fenómeno a que hace referencia”. (pp. 97-98)

\section{¿EXISTE La CONSTRUCCIÓN DE LOS DERECHOS SOCIALES?}

Desde la función de Estado garante, se ha olvidado que la condición de interno no limita el ejercicio de los derechos sociales y que incluso hacerlo —como es el caso del sistema penitenciario mexicano- perjudica en mayor medida no solo a quien padece el internamiento, sino, en lo específico, a las personas (familia) que dependen económica, social y emocionalmente de este. Después de todo, detrás de los que ahora conocemos como "derechos sociales” está la reivindicación de la legítima aspiración — primero social y política— de seres humanos concretos a tener condiciones de vida digna, que suele ser negada por factores estructurales de poder. Nunca está de más recordar, con Bobbio, que el derecho y el poder son las dos caras de una misma moneda (Gutiérrez Rivas y Salazar Ugarte, 2011, p. 13). Desde este mirador se entiende que los derechos sociales, como la mayoría de los derechos humanos, son producto de acciones políticas que solo hasta un segundo momento se transformarían en normas, y que, con el paso del tiempo, se irían ampliando hacia las personas que serían sus titulares (en el extremo, todas ellas) y hacia los bienes protegidos (a las reivindicaciones laborales originales se sumarían, a partir del constitucionalismo de posguerra ya entrado el siglo xx, la salud, la vivienda y la educación, entre otros).

Avanzar en la consolidación de los derechos más fundamentales de las personas privadas de la libertad es el gran reto de los Estados latinoamericanos. Difícil es siquiera pensar que la discusión se pueda centrar en el reconocimiento y asignación de derechos del orden social cuando apenas si se piensa en los derechos a la vida, a la dignidad o a la salud. El 
presente artículo pretende — si acaso, apenas sí propone- centrar el debate en la posibilidad de reconocer la construcción de los derechos sociales en las personas privadas de la libertad; en qué momento es posible su asignación y derecho legítimo cuando la construcción de los derechos en sí mismos es todavía una cuenta pendiente.

La gran mayoría de legislaciones y garantías constitucionales tendientes a establecer los principios o bases del sistema penitenciario de cada Estado concuerdan con que la imposición de penas y medidas de seguridad es un asunto de monopolio estatal, cuya atribución recae exclusivamente en las autoridades sustantivas de la materia. Asimismo, el orden de la ejecución penal puede establecerse a partir de la jerarquía estatal (municipio, estado y nación, en el caso de México); pero también se concuerda con que el establecimiento de penas suprime de forma temporal la libertad y el ejercicio pleno de los derechos civiles y políticos.

Los derechos políticos son toda expresión tangible de la vitalidad de las sociedades, toda vez que, desde su ejercicio, tanto individuos como grupos sociales pueden construir acuerdos para elegir a quienes los representen y a quienes los gobiernen. Su práctica puede marcar el sentido de las acciones legislativas y gubernativas, con el propósito de que cumplan y atiendan expectativas y necesidades, en los órdenes materiales y culturales necesarios para la vida colectiva. Luigi Ferrajoli (2001) establece que la democracia se construye siguiendo cuatro vertientes que corresponden a los cuatro tipos de derechos humanos: 1) derechos políticos que comprende la democracia política, 2) derechos civiles en cuanto a la democracia civil, 3) derechos de libertad en cuanto a la democracia liberal y 4) derechos sociales en cuanto a la democracia social (pp. 160-162).

El reconocimiento de los derechos civiles y políticos está estrechamente relacionado con el ejercicio de la democracia social, y con ello corresponde entonces abordar los derechos sociales como prerrogativas inherentes a la condición de persona, no solo de ciudadano. Esto porque desde el punto de vista teórico no hay ninguna incompatibilidad entre la agenda de los derechos sociales y el proyecto político liberal que se propone limitar el poder con la finalidad de ofrecer garantías a las libertades fundamentales. Las constituciones contemporáneas contemplan de manera simultánea en el catálogo de derechos fundamentales tanto los derechos sociales como los civiles y las libertades básicas, junto con los derechos políticos de tradición y proyección democrática y otros derechos fundamentales, como el derecho a la no discriminación (Gutiérrez Rivas y Salazar Ugarte, 2011, p. 18). 
Sin embargo, habría que tomar esta oportunidad para distinguir entre los derechos sociales — también conocidos como derechos económicos, sociales y culturales (DESC) — y los llamados derechos civiles y políticos, de todos los cuales, estos últimos consisten, como señala Cruz Parcero (2012), "en obligaciones de abstención por parte del Estado o de ciertos particulares, mientras que los derechos sociales implican obligaciones de hacer o dar algo; los primeros son, pues, derechos pasivos y los segundos, derechos activos" (pp. 73-74). También cabe aclarar que la intención del presente artículo no es confundir ni, mucho menos, igualar la consecución de los primeros con los segundos según una misma naturaleza, sino, por el contrario, establecer la idea de que las personas privadas de la libertad tienen derecho a acceder a ambos (derechos pasivos y activos) y que, frente a ellos, el Estado tiene — adquiere - obligaciones tanto de hacer como de no hacer. Como lo establece Cruz Parcero cuando menciona que "a los derechos civiles y políticos les corresponden obligaciones de no hacer (abstenciones u obligaciones negativas), mientras que a los derechos sociales les corresponden obligaciones de hacer (obligaciones positivas)" (p. 76).

En ese sentido, según la Oficina del Alto Comisionado de las Naciones Unidas (ACNUDH) (2009), "una violación de los derechos económicos, sociales y culturales [entendiendo estructuralmente los DESC como derechos sociales] tiene lugar cuando un Estado incumple su obligación de garantizar que tales derechos se disfruten sin discriminación o su obligación de respetarlos, protegerlos y realizarlos" (p. 22). Señala, además, que algunos ejemplos de violaciones de los derechos económicos, sociales y culturales incluyen:

- El desalojo forzoso de personas de sus hogares (derecho a una vivienda adecuada);

- La contaminación del agua, por ejemplo, con desechos de instalaciones de propiedad del Estado (derecho a la salud);

- El hecho de que no se garantice un salario mínimo suficiente para llevar una vida digna (derecho al trabajo);

- El hecho de no evitar el hambre en todas las zonas y comunidades del país (derecho a la protección contra el hambre); 
- La denegación de acceso a la información o los servicios relacionados con la salud sexual y reproductiva (derecho a la salud);

- La segregación sistemática de los niños con discapacidad de las escuelas generales (derecho a la educación);

- El hecho de no impedir que los empleadores lleven a cabo discriminaciones en la contratación (por razones de sexo, discapacidad, raza, opinión política, origen social, situación en cuanto al viH, etc.) (derecho al trabajo);

- El hecho de no prohibir que las entidades públicas o privadas destruyan o contaminen los alimentos y sus fuentes, así como los suelos arables y los recursos hídricos (derecho a la alimentación);

- El hecho de no establecer una limitación razonable de las horas de trabajo en los sectores público y privado (derecho al trabajo);

- La prohibición de utilizar los idiomas minoritarios o indígenas (derecho a participar en la vida cultural);

- La denegación de la asistencia social a personas a causa de su condición (por ejemplo, las personas sin un domicilio fijo o las que solicitan asilo) (derecho a la seguridad social);

- El hecho de no garantizar una licencia de maternidad a las mujeres trabajadoras (protección de la familia y asistencia a esta);

- La desconexión arbitraria e ilegal de la red de abastecimiento de agua para uso personal y doméstico (derecho al agua). (pp. 22-23)

En términos específicos, los derechos sociales a los cuales las personas privadas de la libertad pudieran tener acceso son, sin duda, los relacionados con la condición mínima de alimentación, salud y empleo. En múltiples casos, como ya se ha señalado, los Estados reconocen normativamente que la condición de pena suspende de forma permanente el ejercicio de los 
derechos civiles y políticos: derecho a votar y ser elegido, libertad de expresión, acceso a la información, libertad de asociación y de reunión, libre circulación y residencia, nacionalidad y asilo, derechos humanos en tiempos de guerra, derecho humano a la paz; por su parte, el Pacto Internacional de Derechos Civiles y Políticos reconoce, además, como derechos civiles y políticos los asociados a la condición de igualdad frente al goce de derechos como estos: el derecho a la vida; la prohibición de tortura, penas o tratos crueles, inhumanos o degradantes; la prohibición de que los presos ejecuten trabajos forzosos o degradantes, además del derecho a la reparación, la separación entre presos, la reforma social y la libertad de pensamiento, expresión y asociación, así como el de la no discriminación, entre otros.

Como es posible observar, gran parte de los derechos reconocidos por los DESC representan las acciones que pueden configurar violaciones a la condición mínima de las personas privadas de la libertad. Negar tal afirmación significaría reconocer que las penas y medidas de seguridad disminuyen al mínimo la condición de persona, sin importar cualesquiera que sean los tipos penales y las medidas de seguridad impuestas, no solo en su condición de ciudadano debido a la disminución (suspensión) de sus ejercicios políticos frente al Estado, sino frente a su condición misma de persona.

La mismo onU afirma que frecuentemente una violación de tales derechos guarda relación con la violación de otros derechos; es decir, la violación de tan solo uno de los derechos sociales, civiles o políticos significa la violación sistemática de otros derechos de igual o mayor condición. Con ello, se terminaría configurando sin duda alguna la representación de la calidad de grupo vulnerable para las personas privadas de la libertad.

\section{UN SISTEMA EN CRISIS Y LA URGENTE NECESIDAD DE RECONOCER}

\section{LOS DERECHOS DE LAS PERSONAS PRIVADAS DE LA LIBERTAD}

Es imposible no aseverar que los sistemas carcelarios de América Latina se encuentran en crisis: el aumento de la incidencia delictiva y la falta de mecanismos de gobernanza tanto local como global, pero también el aumento de la desigualdad social, la pobreza y la falta de oportunidades salariales y educativas han posibilitado el engrosamiento de las cárceles, lo que ha representado para miles de personas encontrar en las rejas alguna forma de vida 
a cambio de la promesa de concretar una restitución social. Las cárceles han pasado de representar ese castigo ejemplar que buscaba el Estado a simplemente albergar personas. En prisiones como las del Estado mexicano, los carteles y los grupos de autogobierno controlan todo lo que pasa dentro de los establecimientos y se encargan de seguir administrando la comisión de delitos, como el secuestro o la extorsión. El problema carcelario no solo se limita a las restricciones que tiene el sistema, sino también a las inobservancias y omisiones que suceden estrictamente en materia de derechos humanos (Huertas, Leyva, Lugo, Perdomo y Silvero, 2016).

La vida en prisión se vuelve destructiva, debido a que no se reducen al máximo los efectos nocivos del internamiento, como son el de prisionalización, despersonalización, cambio de conducta, agresividad, depresión y abandono familiar, sino que, por el contrario, se acentúan los nexos entre los grupos delictivos y se somete a la población confinada acondiciones infrahumanas, con falta de asistencia médica y de higiene y bajo la nula o poca supervisión de las autoridades penitenciarias. Se elimina, además, toda posibilidad de favorecer más los vínculos sociales, la colaboración y participación de las entidades públicas y privadas y el acceso a las prestaciones públicas.

Sin embargo, no son únicamente las condiciones latentes lo que significa la vida en prisión, lo son también la restricción del ejercicio de los derechos que no quedan suspendidos con la privación de la libertad, que, en sentido estricto, corresponden de forma exclusiva al ejercicio de los derechos civiles y políticos. ¿Pero qué pasa entonces con el ejercicio de los derechos sociales? Son estos los mismos que las constituciones políticas reconocen y que en México se consolidan a través del artículo 18 constitucional, cuyo reconocimiento no es limitativo, sino que, por el contrario, se presume su garantía en cuanto a la educación, la salud, el deporte y el derecho al trabajo.

La Comisión Nacional de Derechos Humanos señala, a través de su Informe Anual de actividades 2019, que por medio del Diagnóstico Nacional de Supervisión Penitenciaria (DNSP) se ha podido identificar que, tan solo en México,

[...] existe una gran insuficiencia de actividades laborales, educativas y deportivas, así como de capacitación para el trabajo, además de que, la deficiencia en la atención de la salud de las personas en reclusión continúa 
siendo, en la generalidad de los centros, una problemática mayor, ocasionada tanto por la falta de personal médico como de equipo y fármacos necesarios para atención de la salud. (Comisión Nacional de Derechos Humanos, 2019)

Pero no solo eso; también se señala, en el apartado sobre el análisis situacional de los derechos humanos de las personas privadas de la libertad del mismo informe, que

[...] durante los últimos ańos, se encuentra: la falta de separación entre procesados y sentenciados $(77.8 \%)$, deficientes condiciones materiales y de higiene en diversas instalaciones de los centros (76.3\%), el hacinamiento (43.8\%), la sobrepoblación (32\%), insuficiente personal médico, de seguridad y custodia $(68.7 \%)$, deficiencia en la atención al derecho de protección de la salud (48.8\%); así como, la presencia de condiciones de autogobierno y violencia al interior de los establecimientos penitenciarios (49.6\%). (Comisión Nacional de Derechos Humanos, 2019)

En otro escenario, el caso de Colombia es otro ejemplo que configura la situación de crisis que somete en una condición de vulnerabilidad a miles de presos en sus cárceles. La Corte Constitucional colombiana ha diseñado, desde 1998, una línea jurisprudencial compuesta por las sentencias T-153-98, T-256-00, T-388-13, T-815-13, T-861-13 y T-762-15, en las que advierte sobre una crisis que pone en riesgo la integridad y vida de las personas. Esta problemática motivó al alto tribunal mediante esos fallos a declarar el estado de cosas inconstitucional en el sistema penitenciario y carcelario (Huertas Díaz, 2020). Y no hay que olvidar la terrible crisis que viven países centroamericanos como Guatemala, Honduras o El Salvador, donde el predominio de la incidencia delictiva se ve enmarcado por el gran número de miembros de pandillas que se encuentran tras las rejas. En esos países no ha quedado otra opción que "encerrar y hacinar" a quienes no tienen garantía de resocialización ni de no repetición, que se amotinan en cárceles bajo condiciones mínimas de estancia y encarcelamiento.

Pero no son tampoco estos los únicos casos; el Instituto Latinoamericano de las Naciones Unidas para la Prevención del Delito y el Tratamiento del Delincuente, en su reciente estudio sobre la situación del covid-19 publicado en abril del 2020, refiere que la mayoría de los Estados latinoamericanos padecen condiciones de hacinamiento carcelario (tabla 1), 
lo que significa que gran parte de sus centros de reclusión se encuentran rebasados en su capacidad de internamiento tanto para condenados como para sentenciados (Chavarría Durán, 2020, p. 7). El problema de padecer hacinamiento (sobrepoblación carcelaria) radica en que estructuralmente esta situación no solo obliga a los internos a padecer una estricta convivencia sujeta a limitaciones en el espacio y el acceso a bienes de consumo vital, como alimentos, agua y productos de primera necesidad, así como servicios mínimos de salud e higiene, sino que también configura violaciones sistemáticas de derechos fundamentales, al tiempo que los somete a una condición de vulnerabilidad.

Tabla 1. Sobrepoblación penitenciaria en América Latina

\begin{tabular}{|lcccc|}
\multicolumn{1}{r}{ País } & Ańo & Capacidad del sistema & Población existente & Exceso \\
\hline Uruguay & 2018 & 11887 & 10098 & -1789 \\
\hline México & 2018 & 215825 & 203847 & -11978 \\
\hline Chile & 2018 & 41826 & 43089 & 1263 \\
\hline Argentina & 2016 & 67110 & 76261 & 9151 \\
\hline Panamá & 2018 & 14830 & 17064 & 2234 \\
\hline Ecuador & 2018 & 27270 & 37530 & 10260 \\
\hline Costa Rica & 2018 & 9925 & 13833 & 3908 \\
\hline Brasil & 2018 & 409948 & 798753 & 298805 \\
\hline Colombia & 2018 & 79723 & 121230 & 41507 \\
\hline
\end{tabular}

Fuente: Elaboración propia con datos del Instituto Latinoamericano de las Naciones Unidas (Ilanud), 2020.

Ese mismo informe destaca:

El de lo penitenciario es uno de los ámbitos en los que es más importante partir de presupuestos como los indicados, en vista de que la situación de las personas privadas de libertad les ubica en un medio harto propenso a sufrir los efectos de todas aquellas vulnerabilidades a las que se ha hecho ya referencia, producto a su vez de la exclusión social característica, casi siempre, de estas personas. (2020, p. 5) 
Puede afirmarse, por tanto, que 1) las personas privadas legalmente de la libertad sí pueden ser consideradas como un grupo vulnerable, 2) las condiciones de encarcelamiento que padecen miles de personas en América Latina violentan de forma sistemática sus derechos más fundamentales y, 3) los derechos sociales son de posible alcance para este grupo, siempre y cuando se considere a priori su reconocimiento.

No se debe nunca olvidar que la construcción de los derechos humanos a lo largo de la historia ha correspondido a una incansable lucha de los más débiles sobre los más fuertes. Nunca antes un derecho ha sido reconocido por la mera voluntad de quienes ostentan el poder, quienes, en su momento tuvieron la oportunidad $-\mathrm{y}$ podían hacerlo- de conceder una serie de libertades y prerrogativas a petición de la colectividad, y, más aun, de los que ahora se reconocen como grupos vulnerables o de la inmensa cantidad de grupos de minorías que hoy en día están presentes en los diversos grupos y estatus sociales. Como lo señalan especialmente Gutiérrez Rivas y Salazar Ugarte (2011),

[...] tiene un gran significado el hecho de que los derechos sociales, como la mayoría de los derechos, desde siempre han sido los "derechos del débil" frente a los fuertes $[\ldots]$ en particular los de este tipo, son expresiones de reivindicaciones que parten de una situación injusta y opresora y que aspiran a transformarla. (p. 20)

¿Entonces por qué no pensar en la construcción de los derechos sociales desde la privación de la libertad? La respuesta seguramente tendría que centrarse en la incompetencia estatal; sin embargo, cuando se habla de derechos subjetivos, se piensa que la tarea está dada únicamente en lo que el Estado hace desde la trinchera de la institucionalidad y, en lo específico, desde el aparato jurídico-institucional, olvidando que parte de la tarea radica en el reconocimiento pleno que sobre dicha medida o condición se tendría que enunciar. Pero acá volvemos al tema que abordamos apenas de forma breve al inicio de este artículo: los penados no representan un trabajo de reforma que gane adeptos y simpatizantes dentro del conglomerado social, sino que, por el contrario, resta simpatía en cuanto a los "privilegios" que, para muchos, dentro del vulgo social, no deberían de tener (disfrutar) los que delinquen, ya que buscan irremediablemente penas y medidas cada vez más duras, sin dejar de considerar la prisión como la reina de las penas. 
Es entonces cuando el reconocimiento de los derechos de las personas privadas de la libertad se reduce a la posibilidad de que el gobierno o la legislación en turno permita, en contra incluso de la mayoría social, que se creen mecanismos idóneos para brindar recursos jurídicos, económicos y, en especial, políticos para que cesen las condiciones de vulneración y sistemática violación de derechos. Ocurre lo mismo con el pronunciamiento sobre el reconocimiento de derechos más allá de lo que establece el principio constitucional, en cuanto el artículo 1 de la Constitución mexicana señala que "todas las personas gozarán de los derechos humanos reconocidos en esta Constitución y en los tratados internacionales de los que el Estado Mexicano sea parte, así como de las garantías para su protección, cuyo ejercicio no podrá restringirse ni suspenderse, salvo en los casos y bajo las condiciones que esta Constitución establece".

Cruz Parcero (2012) menciona, siguiendo a Robert Alexy, que se debe entender que los derechos humanos son esencialmente principios "principios constitucionales" (pp. 8990), que están de forma abstracta, razón por la que no se dice cuáles son las condiciones de aplicación; es decir, los derechos subjetivos están plenamente reconocidos en los instrumentos normativos — dígase Constitución—; sin embargo, no se establecen las reglas de su cumplimiento ni se especifica en qué condiciones o en cuáles circunstancias se tienen que dar estos. De ahí que, durante el tiempo en que los sistemas jurídicos latinoamericanos se rigieron principalmente a través del modelo positivista, existieron miles de violaciones de derechos humanos de toda naturaleza, pues el positivismo no reconocía como derechos en sí mismos los derechos subjetivos, sino que más bien estos eran considerados como normas inacabadas. Se suponía que estas no establecían condiciones de aplicación ni, mucho menos, sanciones, lo que daba lugar a que, ante una interrogante o una laguna en la aplicación de los derechos subjetivos, "operara la discrecionalidad de los jueces, la misma que se empleaba de forma imprecisa y sin certeza jurídica, pues en estos casos las reglas eran todavía vagas y la norma no previa todos los casos específicos" (Rodríguez, 2016, pp. 11-37).

Pero ahora, a través del modelo garantista, existe una certeza centrada en el principio pro persona, que exige que la interpretación normativa deba ser aplicable en el sentido más favorable a la persona y menos restrictiva en cuanto al ejercicio y respeto de sus derechos. En el caso de México, dicho reconocimiento vino a partir de la reforma del 2011 en materia de derechos humanos, cuando en el artículo 1 constitucional se reconoció la aplicación de los 
derechos humanos y se aplicó en esencia el principio pro persona para interpretar los derechos de acuerdo con el control de convencionalidad. Por su parte, en el caso de Colombia, en el artículo 93 de su Constitución, estos derechos — reconocidos en tratados que prohíben su limitación en estados de excepción — prevalecen en el orden interno. A su vez, la Corte Constitucional colombiana, en los primeros pasos que dio hacia la construcción del concepto de "bloque de constitucionalidad", consideró justamente la primera parte del artículo de referencia; fue después cuando resolvió que incluía también los tratados internacionales de derechos humanos y de derecho humanitario (C. C., Sentencia T-1319/01, Colom.).

La importancia de incorporar la convencionalidad a través del contenido de los tratados internacionales dentro de las decisiones judiciales en el derecho interno resulta de que, aunque se realice de forma tácita el reconocimiento de los derechos subjetivos en las normas constitucionales, su aplicación resulta inocua en muchas ocasiones, al no determinar como se ha señalado - las reglas de aplicación y de protección de los derechos reconocidos. En muchas ocasiones esto dificulta su tutela de forma efectiva; sin embargo, los tratados sí establecen reglas específicas para aplicar la protección de los derechos; de ahí la importancia de adherirlos a partir del bloque de constitucionalidad que garantiza la aplicación del derecho más favorable (Daza, 2013).

Frente a ello, en lo concerniente al sistema penitenciario mexicano, puede establecerse que, a pesar de todo, la premisa constitucional reconoce como base del sistema carcelario los derechos humanos y la incorporación de la educación, la salud, el deporte y el trabajo como máximas del proceso resocializador. En nada considera la limitación de las acciones de política criminal que reducen al mínimo las condiciones de internamiento, de acuerdo con lo que establecen los instrumentos internacionales de la materia. Se cuenta entonces con un sistema que reconoce derechos de difícil alcance, pues no limita el cese en el ejercicio de las acciones que propician la violación sistemática de derechos dentro de las prisiones (Gamboa, 2016).

\section{Conclusiones}

A partir del presente artículo, se ha podido establecer de forma prioritaria que la identificación y construcción de los derechos sociales aplicables al derecho ejecutivo penal 
en América Latina obedece a una tradición jurídica que omite y restringe la aplicación efectiva de los derechos de las personas privadas de la libertad. No solo se trata de las violaciones en materia de derechos humanos o de su inobservancia, sino de la omisión normativa de todos los derechos que no son privativos de la conducta antijurídica, pero que no corresponden a una aplicación práctica que coadyuve, además, a configurar el cumplimiento de la función ius puniendi del Estado y de la pena en sí misma, cual es la reinserción social.

Creo justificadamente que el sistema penitenciario en América Latina atraviesa uno de los momentos más críticos de la historia moderna, al mantener condiciones de verdadera crisis: el hacinamiento, el autogobierno, la extorsión, las condiciones insalubres, las deficiencias de alimentación, la falta de atención médica, la restricción del ejercicio de derechos de petición, los sustitutivos penales, así como tendría que revisarse la concesión de cierto tipo de beneficios o condiciones indispensables para el internamiento de los distintos grupos que integran la población penitenciaria y que se restringen al otorgamiento de los beneficios preliberacionales, la necesidad de espacios seguros para la población LGBTTTIQ, el derecho a la maternidad y la lactancia, el derecho a la educación, salud y el empleo, entre muchos otros derechos, todo lo cual impone sobre la población interna condiciones de vulnerabilidad y que, al mismo tiempo, configuran obligaciones imposibles de cumplir para el Estado.

La posibilidad de reconocer el cumplimiento de los derechos fundamentales de las personas privadas de la libertad, en el ejercicio de sus derechos sociales, permitiría coadyuvar a la consolidación del proceso de reinserción social o de reeducación que corresponden al propósito que tiene la imposición de la pena. Es imposible pretender que una persona pueda reinsertarse adecuadamente en una sociedad que la ha estigmatizado y que la segrega situándola en el grupo de los indeseables, luego de haber pasado años dentro de una prisión y sin que, además, tenga los incentivos prestacionales que dejo de "tener" por estar privada de la libertad.

Una persona con antecedentes penales, sin posibilidad de acceder a prestaciones educativas, salariales o culturales, es exponencialmente una persona que seguramente tendrá que remitir sus esfuerzos de reinsertarse laboral y socialmente a través del trabajo informal y, en el peor de los casos, podrá optar por reincidir en conductas de tipo penal cada vez más 
graves. Es altamente probable que no encuentre en ese entorno social la posibilidad de obtener mecanismos para el efectivo ejercicio de sus derechos, que quedaron suspendidos, primero, de forma temporal y luego, de forma permanente tras la comisión de un delito, sin importar la gravedad y naturaleza de este.

El reconocimiento de los derechos sociales de las personas privadas de la libertad es todavía una construcción en proceso que requiere de la voluntad estatal e institucional, que debe permitir no solo la incorporación sino la creación de mecanismos jurídico-institucionales fehacientes para garantizar su respeto irrestricto y no la pretensión de incorporar políticas que nunca tendrán un efecto práctico ni real que constituya su reconocimiento. Desafortunadamente, América Latina exhibe una profunda tradición de violación sistemática de los derechos de las personas privadas de le libertad, de modo que las cárceles se han convertido en auténticas máquinas de aplastamiento donde se vive todos los días "la ley del más fuerte". Se espera que la producción de trabajos como este pueda propiciar condiciones para que los derechos de estos grupos puedan ser salvaguardados.

\section{REFERENCIAS}

Alexy, R. (1993). Teoría de los derechos fundamentales. Madrid: Centro de Estudios Constitucionales.

Bobbio, N. (1991). El tiempo de los derechos. Madrid: Sistema.

Chavarría Durán, D. (2020). El sistema penitenciario ante la encrucijada producto de la crisis provocada por el covid-19. Recuperado de http://www.ilanud.or.cr/wp-content/ uploads/2020/04/ILANUD-COVID-19.pdf?fbclid=IwAR3qnt_un5RVjgAQG53IYOBNPvJotHt4TVny1mT1LdfK2cDvvL-Srydv0KM\%0A\%0A

Comisión Nacional de Derechos Humanos. (2019). Informe anual de actividades 2019. Recuperado de http://informe.cndh.org.mx/menu.aspx?id=30087

Corte Constitucional de Colombia [C. C.]. (2001). M. P. Rodrigo Uprimny Yepes. Sentencia T-1319/01. Bloque de constitucionalidad. Evolución jurisprudencial.

Cruz Parcero, J. A. (2000). Los derechos sociales como técnica de protección. Ciudad de México: Universidad Nacional Autónoma de México. 
Cruz Parcero, J. A. (2006). Los métodos para los juristas. En C. Courtis (Ed.), Ensayos sobre la metodología de la investigación jurídica. Madrid: Trotta.

Cruz Parcero, J. A. (2012). El lenguaje de los derechos. Ciudad de México: Trotta.

Daza, A. (2013). Legalidad y prescripción frente a la investigación de crímenes de lesa humanidad en Colombia. IUSTA, 38(1), 205-223.

Ferrajoli, L. (2001). Derechos y garantías. La ley del más débil. Madrid: Trotta.

Gamboa, S. (2016). A propósito de una verdad contrahegemónica: aprendizajes desde la decisión de la Corte Penal Internacional sobre el ataque contra la flotilla de la libertad. Via Inveniendi et Iudicandi, 11(1), 11-35. Recuperado de http://revistas.usantotomas. edu.co/index.php/viei/article/view/2925/2797

Gutiérrez Rivas, R. y Salazar Ugarte, P. (2011). Igualdad, no discriminación y derechos sociales: una vinculación virtuosa. Ciudad de México: Consejo Nacional para Prevenir la Discriminación.

Hernández Sampieri, R., Fernández Collado, C. y Baptista Lucio, P. (2014). Metodología de la investigación. Ciudad de México: McGraw Hill.

Hervada, J. (2000). Introducción crítica al derecho natural. Pamplona: Ediciones Universidad de Navarra (EUNSA).

Huertas Díaz, O. (2019). Politica criminal sistémica: origen rizomático y contribuciones para su reflexión. Bogotá: Grupo Editorial Ibáñez.

Huertas Díaz, O. (2020). Liberar detenidos y condenados: entre la crisis penitenciaria y el covid-19. UN Periódico Digital. Recuperado de https://unperiodico.unal.edu.co/pages/ detail/liberar-a-detenidos-y-condenados-entre-la-crisis-penitenciaria-y-el-covid-19/

Huertas, O., Leyva, M., Lugo, L., Perdomo, W. y Silvero, A. (2016). Entre la minimización y la expansión del derecho penal: la presencia de Beccaria en el debate contemporáneo. IUSTA, 44(1).

Oficina del Alto Comisionado de las Naciones Unidas para los Derechos Humanos (ACNUDH). (2009). Preguntas frecuentes sobre los derechos económicos, sociales y culturales (No. Folleto informativo 33). Ginebra. Recuperado de https:/www.ohchr.org/Documents/ Issues/ESCR/FAQ on ESCR-sp.pdf 
Organización de las Naciones Unidas (onu). (2020). Pacto Internacional de Derechos Económicos, Sociales y Culturales. Adoptado y abierto a la firma, ratificación y adhesión por la Asamblea General en su resolución 2200 A. Recuperado de https://www.ohchr.org/ $\mathrm{sp} /$ professionalinterest/pages/cescr.aspx

Rodríguez, E. (2016). El pasaje del Estado y el derecho a la postmodernidad. Via Inveniendi et Iudicandi, 11(2), 11-37.

Sánchez Zorrilla, M. (2011). La metodología en la investigación jurídica: características peculiares y pautas generales para investigar en el derecho. Revista Telemática de Filosofía Del Derecho, (14), 317-358. Recuperado de http://www.rtfd.es/numero14/11-14.pdf

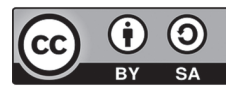

